\title{
Glabridin and Anti-Non-Muscle Myosin IIA Therapy Disrupts Non-Small Cell Lung Carcinoma Motility
}

\author{
Marie Kelly-Worden*, Amy Troesch, Sarah Pruitt, Ryan Rhodes, Deavin Eviston \\ Biology Department, Ball State University, Muncie, Indiana \\ Email: *mlkellyworde@bsu.edu
}

How to cite this paper: Kelly-Worden, M., Troesch, A., Pruitt, S., Rhodes, R. and Eviston, D. (2021) Glabridin and Anti-Non-Muscle Myosin IIA Therapy Disrupts Non-Small Cell Lung Carcinoma Motility. Advances in Lung Cancer, 10, 11-19.

https://doi.org/10.4236/alc.2021.102002

Received: April 21, 2021

Accepted: June 15, 2021

Published: June 18, 2021

Copyright (อ 2021 by author(s) and Scientific Research Publishing Inc. This work is licensed under the Creative Commons Attribution International License (CC BY 4.0).

http://creativecommons.org/licenses/by/4.0/

\section{(c) (i) Open Access}

\begin{abstract}
Lung cancer is the leading cause of cancer related death in the United States killing over 130,000 people each year. While a combination of chemo and radiation therapy may be effective, surgery is still required for many patients. Without surgery, the disease may progress and lead to metastases. We sought to determine if treatment with anti-non-muscle myosin IIA antibody would inhibit movement of the cells in the presence and absence of glabridin (an isoflavonoid compound shown to inhibit cell migration by inhibiting myosin). We compared inhibition by glabridin to that of an anti-non-muscle myosin IIA antibody and a combination therapy of both at 12 and 24 hours post wound creation. Cells that took up the anti-non-muscle myosin IIA antibody were greatly inhibited in motility and exhibited no significant change in wound healing. Glabridin treatment resulted in a dramatic increase in wound size within 12 hours and regeneration within 24 hours. The greatest decrease in motility was observed in cells treated with the combination of both glabridin and anti-non-muscle myosin IIA antibody. By $24 \mathrm{hrs,} \mathrm{cell} \mathrm{mi-}$ gration had halted due to death of the cells resulting from this combination. Further testing needs to be done to determine a safe mode of delivery of the combination therapy to ensure only local distribution. Controlled release drug delivery depot systems have been used as a means to provide local release of drugs intra-tumorally or adjacent to the cancerous tissue after surgical resection and have great potential.
\end{abstract}

\section{Keywords}

Anti-Non-Muscle Myosin IIA Antibody, Cell Migration, Glabridin, Non-Small Cell Lung Carcinoma, Wound Healing Assay 


\section{Introduction}

Each year, over 130,000 people die due to the effects of lung cancer (American Cancer Society). Primary lung cancer is the second most common type of malignancy and ranked first in mortalities correlated with malignancy. Non-small cell lung carcinoma (NSCLC) represents $85 \%$ of lung cancers with $70 \%$ being present with locally advanced or metastatic disease (American Cancer Society) [1] [2] [3] [4] [5]. Multiple genetic as well as microenvironmental factors may effect carcinogenesis and tumor growth of NSCLC including glucose transporters and tumor suppressor factors [6]. Those with NSCLC have a low 5-year survival rate of $15 \%$ [1] [4]. Unfortunately, for years now, surgery has remained the only potentially curative modality for NSCLC; however, approximately $30 \%-55 \%$ of patients develop recurrence even after curative resection. Individual or concurrent chemo or radiation therapy is beneficial in some cases, and in many others these treatments may be used for palliative care [7] [8].

Metastasis is a complicated process involving the interaction of several proteins. For example, HIF- $1 \alpha$ protein synthesis is regulated by activation of the phosphatidylinositol 3-kinase (PI3K) and ERK mitogen-activated protein kinase (MAPK) pathways. Furthermore, these pathways are activated via receptor tyrosine kinases, non-receptor tyrosine kinases, or G-protein-coupled receptors [9] [10].

Fortunately, a new treatment has come to light recently, immunotherapy; the use of medication to help a person's own immune system to more effectively recognize and destroy cancer cells. Just to name a few, these immunotherapy medications include nivolumab, pembrolizumab, and ipilimumab; which act by inhibiting the PD-1/PD-L1 and CTLA-4 pathways (American Cancer Society). $\mathrm{PD}-1$ is a cell surface receptor that functions as a T cell checkpoint. Binding of $\mathrm{PD}-1$ to its ligand, programmed death-ligand 1 (PD-L1), activates downstream signaling pathways and inhibits T cell activation. High PD-L1 expression on tumor cells and antigen-presenting cells mediates tumor immune escape [11] [12]. Another immunotherapy antibody is anti-EGFR (anti-epidermal growth factor receptor) which binds to the extracellular domain of EGFR and disrupts ligand binding as well as the signaling cascade resulting in cell growth inhibition [13] [14].

However, these pathways are not the only immunotherapy targets. The process of metastasis involves the process of cell migration. Cell migration can be viewed as a series of steps involving cell protrusion, front-to-back cell polarization, attachment of the front of the cell to the extracellular matrix via integrins, and finally retraction of the tail of the cell. Nearly all of the steps of migration involve non-myosin muscle II (NM II), which is an actin-binding protein, heavily regulated by the FAK/Src complex that plays a central role in cell adhesions, tissue architecture, as well as cell migration.

Previous studies have indicated that glabridin, an isoflavonoid from licorice root, decreases the active forms of FAK and Src. It enhances the levels of inacti- 
vated phosphorylated Src, which decreases interaction of FAK and Src further [9] [10] [11]. Inhibition of the FAK/Src complex also results in reduced activation of RhoA and myosin light chain phosphorylation [15] [16] [17]. Glabridin has other actions on cells as well. It is known to inhibit cyclooxygenase activity and tends to have an anti-inflammatory effect as well an anti-platlet effect [18]. The compound has antitumor activity that results in a dose-dependant decrease in cell viability of C6 glioma cells [19].

Anti-non-muscle myosin IIA antibody, AM, is a synthetic peptide corresponding to residues in human non-muscle myosin IIA. This subtype of myosin is found in human lung tissue. Therefore, AM reacts with myosin in human lung tissue [20] [21] [22] [23]. We hypothesized that AM binding prevents myosin activation and thus cell migration. We propose that inhibition of movement would be similar to that seen by glabridin addition since inhibition of migration is due to an FAK/Src pathway leading to NM II inhibition.

For this study, we used A549 cells (a line of adenocarcinomic human alveolar basal epithelial cells) as these cells have long been the cell model of choice for laboratory experiments in disease areas such as lung cancer (ATCC). A wound healing assay to test motility was performed. If the wound heals within the $24 \mathrm{hr}$ testing period, a combination treatment of glabridin and AM (here further referred to as GAM) would not be considered as a treatment option for NSCLC due to the recurrence. On the other hand, if the cells have decreased migration or do not grow back at all, this combination treatment should be further tested to determine its effectiveness in the prevention of proliferation of cancer cells compared to glabridin treatment alone.

\section{Materials and Methods}

\subsection{Chemicals}

Glabridin, AM, and Alexa fluor 488 were purchased from Abcam. The glabridin was dissolved in DMSO and stored at $-20^{\circ} \mathrm{C}$ until use. DAPI blue was purchased from Sigma-Aldrich. PBS was purchased from Fisher Scientific.

\subsection{Cell Culture}

The A549 cells (a line of adenocarcinomic human alveolar basal epithelial cells, ATCC) were cultured in RPMI 1640 medium (GE Healthcare Life Sciences) at $37^{\circ} \mathrm{C}$ and split as necessary. A549 cells were seeded in six-well culture plates (three wells per group) with $1 \mathrm{~mL}$ of $1 \times 10^{5}$ cells $/ \mathrm{mL}$ per well and grown to a confluent monolayer over 24 hours in a serum-free medium. Cells were observed under a microscope to ensure a confluent monolayer.

\subsection{Wound Healing Assay}

After the $24 \mathrm{hr}$ incubation period, an artificial lesion was created by scraping each well with a $100 \mu \mathrm{L}$ pipette tip with a ten-minute interval between wells. The wound was washed with PBS. The wells were then separated into four treatment 
groups and run in triplicate. Treatment groups were as follows: 1) control; 2) glabridin $(10 \mathrm{~nm}) ; 3)$ anti-non-muscle myosin IIA antibody (1:250, a concentration within the known binding range); and 4) GAM (10 nM glabridin, 1:250 anti-non-muscle myosin IIA antibody).

DAPI blue was applied to each treatment group at $0 \mathrm{hr}, 12 \mathrm{hr}$ and $24 \mathrm{hr}$ time points. Alexa fluor 488 was applied to visualize anti-non-muscle myosin IIA antibody containing wells. Images were obtained using light and fluorescent microscopy using a Sony DSC-T200 Cybershot camera (8.1 Mp at 5x zoom).

Analysis of the images was accomplished with Image Pro 6 software. Pixel distances were measured between DAPI blue fluorescing cells as well as between Alexa fluor 488 fluorescing cells. Average pixel distances were analyzed using a One-Way ANOVA.

\section{Results}

In this experiment, four groups were tested to determine if glabridin and/or AM treatment would prevent wound healing cell migration when streaked in cell culture. All groups were tested and compared on the basis of cell migration determined by DAPI Blue cell pixel distance across the initial wound and post wound and/or as measured by ALEXA Fluor 488 pixel difference post-wound creation ( 0,12 and 24 hours).

Cell migration observed in the glabridin, AM, and GAM groups all varied according to treatment from control (Figure 1). Cells treated with either glabridin, AM, or GAM all displayed less wound healing than controls over the $24 \mathrm{hr}$ period. At 12 hours, all groups other than the controls displayed an increase in wound pixel distance, with glabridin and GAM displaying the greatest increase in pixel difference. Control cells displayed the least degree of pixel difference between the 0 and $24 \mathrm{hr}$ time points. Recovery was observed at the $24 \mathrm{hr}$ time point for the glabridin (Figure 1, average values, $\mathrm{n}=3$ ). AM displayed virtually no change from the $24 \mathrm{hr}$ time point even with recovery. The combination group, GAM, did not recover in the $24 \mathrm{hr}$ time period as observed with DAPI measurements.

Prevention of wound healing for each group (control, glabridin, AM, and GAM) was then measured using the ALEXA Fluor 488 pixel difference. GAM showed the greatest degree of prevention of wound healing cell migration. This was followed by AM, glabridin, and finally the controls.

This lack of recovery was even more pronounced when examining the AM and GAM groups for the presence of AM antibody (Figure 2). An actual increase in lesion distance was observed in the GAM group by $24 \mathrm{hrs}(\mathrm{p}=2.2 \times$ $\left.10^{-5}\right)$. In fact, GAM treatment resulted in widespread cell death and cell distance had to be capped at 2000 pixels.

Background fluorescence (using fluorescent microscopy) and lesion borders (using light microscopy) were used to determine the distance of control and glabridin only treated wells. Glabridin was seen to minimally prevent cell migration as compared to AM and GAM (Figure 2). 


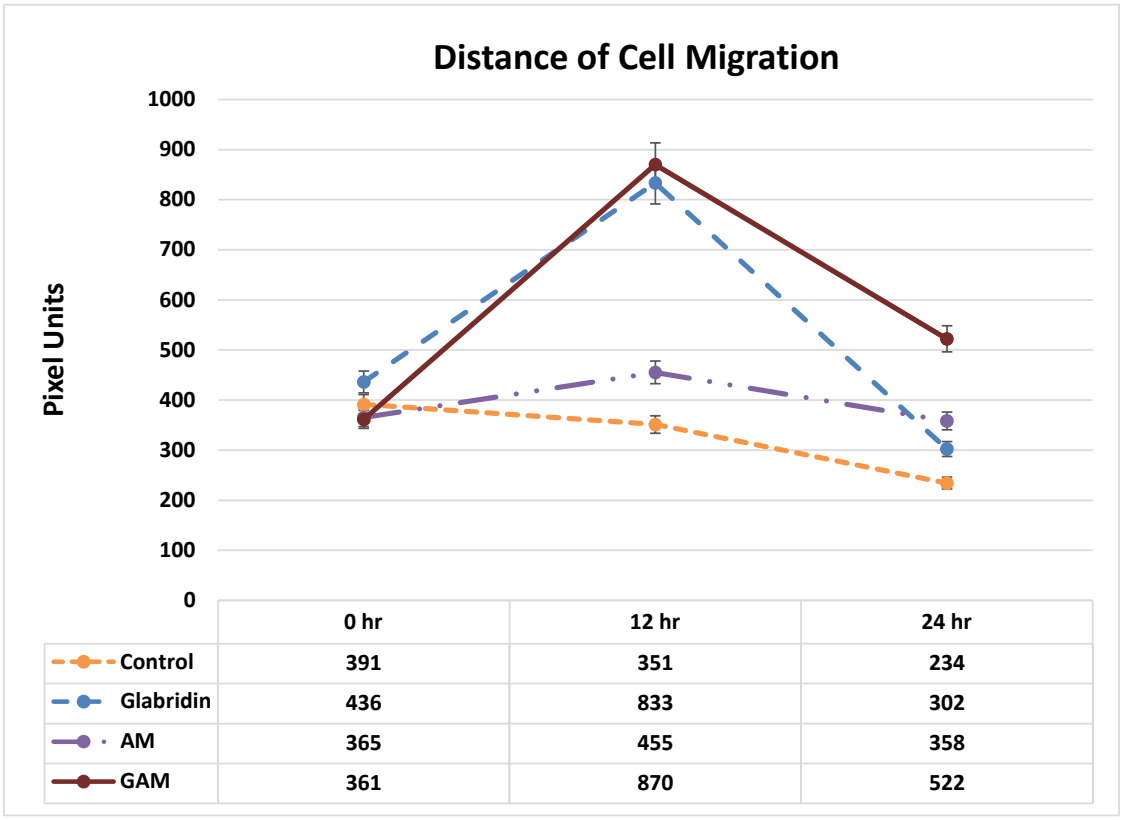

Figure 1. Cell migration as determined by DAPI Blue cell pixel distance. Cell migration for each group (control, glabridin, AM, and GAM) was measured using DAPI blue cell pixel distance at the 0,12 , and $24 \mathrm{hr}$ time points. Cells treated with GAM displayed the least wound healing cell migration followed by AM, glabridin, and finally the controls over the full 24 hour period.

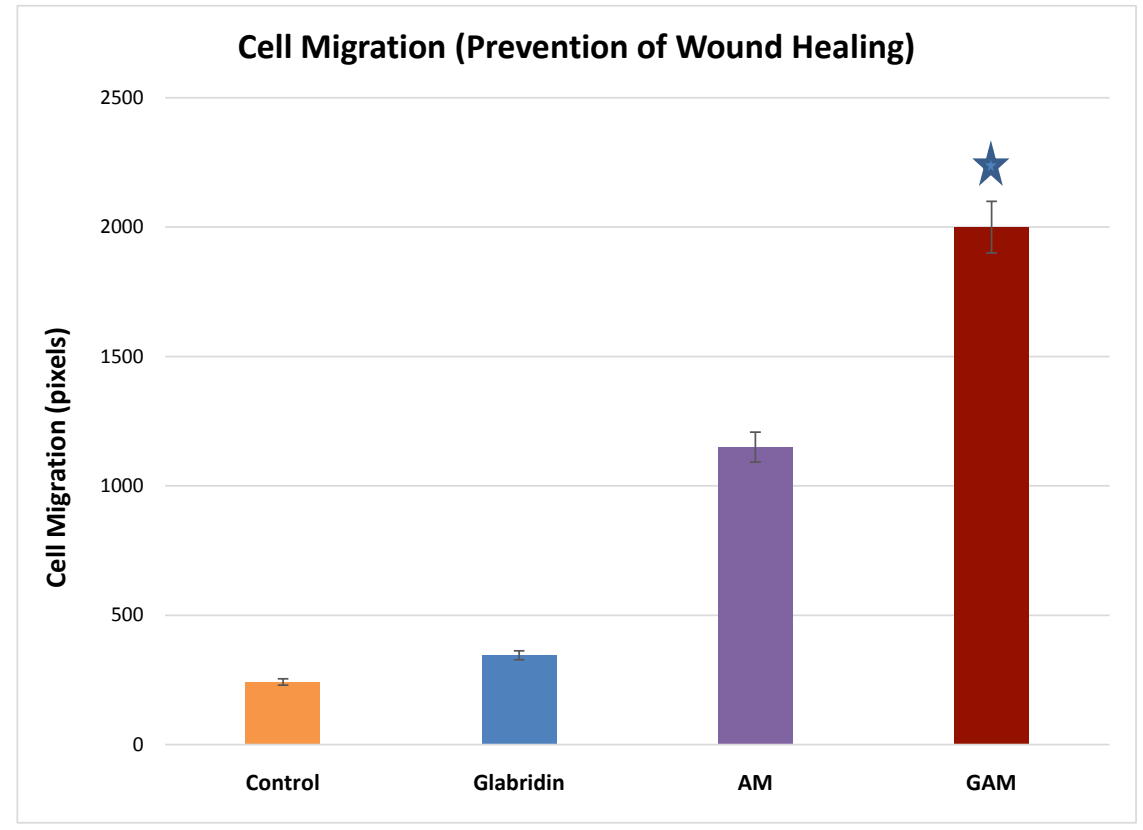

Figure 2. Cell Migration as measured by ALEXA Fluor 488 pixel difference. Prevention of wound healing for each group (control, glabridin, AM, and GAM) was measured by using the ALEXA Fluor 488 pixel difference. GAM showed the greatest degree of prevention of wound healing cell migration followed by AM, glabridin, and finally the controls.

\section{Discussion}

The effectiveness in prevention of wound healing cell migration of cells treated 
with AM, glabridin, and a combination of the two, GAM, was measured in this paper. It was observed that cells treated with AM were significantly inhibited in mobility when compared to cells that were not treated with the AM antibody. This was demonstrated by the presence of green fluorescence using ALEXA Fluor 488 pixel width distance. The ALEXA Fluor 488 dye binds to the AM and produces a fluorescence of the cells treated with AM. The distance between fluorescence measured at 12 hours was found to be slightly larger. This increase in cell width distance is a result of the presence of AM and not due to cell death resulting from initial wound creation. This is verified since controls do not experience this increase in cell width distance. Therefore, cell width distance increase is attributed to AM's prevention of wound healing. AM was also found to have a cell migration distance that remained fairly constant between initial wound width and final wound width (being approximately equal to the original wound distance demonstrated by the blue fluorescence of DNA using DAPI Blue cell pixel width distance). These results were expected as AM binds to myosin, preventing myosin activation, and therefore preventing cell migration. As demonstrated, once the cells were streaked, they had little growth recurrence in the region of the wound showing promise for the use of AM for medicinal treatment of NSCLC.

A decrease in cell motility was also seen by the cells treated with glabridin, although not to the same degree as the cells treated with AM. Cells treated with glabridin displayed even greater wound distances at the 12 hour time point, but had a recovery similar to that of the controls by the 24 hour time point as seen by the DAPI Blue cell pixel width distance. This was also demonstrated by the ALEXA Fluor 488 (ALEXA Fluor 488 generates a background fluorescence when applied to cells that can be distinguished from the specific binding of the antibody) pixel width difference measurements, as prevention of wound healing measured by background green fluorescence was found to be similar to that of the controls. Due to this recovery observed at the $24 \mathrm{hr}$ time point, it can be hypothesized that the cells either underwent apoptosis prior to this time point or migrated away from the wound. The decrease in wound healing migration may be attributed to the fact that glabridin decreases the active forms of FAK and Src, enhances the levels of inactivated phosphorylated Src, which decreases interaction of FAK and Src further. Inhibition of the FAK/Src complex results in reduced activation of RhoA and myosin light chain phosphorylation which further prevents cell migration [9] [10] [11]. However, the increase in wound width may be also due to the antitumor activity of glabridin previously observed in glioma cells [19]. Though glabridin shows promise as a treatment for NSCLC, glabridin by itself may not be the most effective treatment for NSCLC.

Though both AM and glabridin individually reduced cell mobility, prevention of wound healing cell migration was almost twice as large in the cells treated with GAM (glabridin and AM). GAM was found to have the least total cell migration back to its original state as shown by DAPI Blue cell pixel distance, and the greatest prevention of wound healing cell migration as demonstrated by the 
ALEXA Fluor 488 pixel width difference. These results show that there is most likely an additive effect for the combination of AM and glabridin. Though glabridin may decrease migration by affecting the FAK/Src pathway, AM addition provides further inhibition through the binding of myosin. This may prevent escape of the cells from the glabridin treatment and allow for glabridin to exert its cytotoxic effects as well; leading to antitumor activity and the subsequent death of the cells. Thus, apoptosis may have halted migration completely by 24 hours. The use of AM after surgical removal needs to be further investigated using an animal model as well as a combination treatment of glabridin and AM to determine if this treatment would have a further benefit for patients with NSCLC. A delivery system of the combined therapy into the tumor before surgical removal could lead to death of the cells and prevention of spread of the cancer. A subsequent treatment with a gel or film after resection could potentially prevent recurrence of NSCLC.

\section{Conclusion}

Sole AM treatment demonstrated less cell migration and therefore greater prevention of wound healing under our assay conditions than glabridin treatment alone. A combination treatment of both glabridin and AM (GAM) showed the largest degree of prevention of wound healing. Though this therapeutic treatment shows great promise, further testing needs to be done in an animal model to determine potential toxicity as well as a safe mode of delivery of GAM either both or after surgical resection. Controlled release drug delivery depot systems including films, gels, and rods have been used as a means to provide high therapeutic concentrations of chemotherapy by implantation and release of the drug intra-tumorally or adjacent to the cancerous tissue after surgical resection [24] [25] [26]. Once a safe mode of delivery has been determined, this immunotherapy may be used for lung cancer patient treatment.

\section{Conflicts of Interest}

The authors declare no conflicts of interest regarding the publication of this paper.

\section{References}

[1] Lu, T., Yang, X., Huang, Y., et al. (2019) Trends in the Incidence, Treatment, and Survival of Patients with Lung Cancer in the Last Four Decades. Cancer Management and Research, 11, 943-953. https://doi.org/10.2147/CMAR.S187317

[2] Zappa, C. and Mousa, S.A. (2016) Non-Small Cell Lung Cancer: Current Treatment and Future Advances. Translational Lung Cancer Research, 5, 288-300. https://doi.org/10.21037/tlcr.2016.06.07

[3] Lemjabbar-Alaoui, H., Hassan, O.U., Yang, Y.W., et al. (2015) Lung Cancer: Biology and Treatment Options. Biochimica et Biophysica Acta, 1856, 189-210. https://doi.org/10.1016/j.bbcan.2015.08.002

[4] DelaCruz, C.S., Tanoue, L.T. and Matthay, R.A. (2011) Lung Cancer: Epidemiology, 
Etiology, and Prevention. Clinics in Chest Medicine, 32, 605-644. https://doi.org/10.1016/j.ccm.2011.09.001

[5] Molina, J.R., Yang, P., Cassivi, S.D., et al. (2008) Non-Small Cell Lung Cancer: Epidemiology, Risk Factors, Treatment, and Survivorship. Mayo Clinic Proceedings, 83, 584-594. https://doi.org/10.1016/S0025-6196(11)60735-0

[6] Pezzuto, A., D’Ascanio, M., Ricci, A., et al. (2020) Expression and Role of p16 and GLUT1 in Malignant Diseases and Lung Cancer: A Review. Thoracic Cancer, 11, 3060-3070. https://doi.org/10.1111/1759-7714.13651

[7] Uramoto, H. and Tanaka, F. (2014) Recurrence after Surgery in Patients with NSCLC. Translational Lung Cancer Research, 3, 242-249.

[8] National Collaborating Centre for Cancer (UK) (2011) The Diagnosis and Treatment of Lung Cancer (Update). National Collaborating Centre for Cancer (UK), Cardiff.

[9] Semenza, G.L. (2003) Targeting HIF-1 for Cancer Therapy. Nature Reviews Cancer, 3, 721-732. https://doi.org/10.1038/nrc1187

[10] Pezzuto, A., Perrone, G., Orlando, N., et al. (2019) A Close Relationship between HIF- $1 \alpha$ Expression and Bone Metastases in Advanced NSCLC, a Retrospective Analysis. Oncotarget, 10, 7071-7079. https://doi.org/10.18632/oncotarget.27378

[11] Jiang, Y., Chen, M., Nie, H. and Yuan, Y. (2019) PD-1 and PD-L1 in Cancer Immunotherapy: Clinical Implications and Future Considerations. Human Vaccines \& Immunotherapeutics, 15, 1111-1122. https://doi.org/10.1080/21645515.2019.1571892

[12] Redin, E., Garmendia, I., Lozano, T., et al. (2021) SRC Family Kinase (SFK) Inhibitor Dasatinib Improves the Antitumor Activity of Anti-PD-1 in NSCLC Models by Inhibiting Treg Cell Conversion and Proliferation. Journal for ImmunoTherapy of Cancer, 9, e001496. https://doi.org/10.1136/jitc-2020-001496

[13] Vokes, E.E. and Chu, E. (2006) Anti-EGFR Therapies: Clinical Experience in Colorectal, Lung, and Head and Neck Cancers. Oncology, 20, 15-25.

[14] Zhu, K., Li, K., Wang, H., et al. (2019) Discovery of Glabridin as Potent Inhibitor of Epidermal Growth Factor Receptor in SK-BR-3 Cell. Pharmacology, 104, 113-125. https://doi.org/10.1159/000496798

[15] Hsu, Y.L., Wu, L.Y., Hou, M.F., et al. (2011) Glabridin, an Isoflavan from Licorice Root, Inhibits Migration, Invasion and Angiogenesis of MDA-MB-231 Human Breast Adenocarcinoma Cells by Inhibiting Focal Adhesion Kinase/Rho Signaling Pathway. Molecular Nutrition \& Food Research, 55, 318-327. https://doi.org/10.1002/mnfr.201000148

[16] Tsai, Y.M., Yang, C.J., Hsu, Y.L., et al. (2011) Glabridin Inhibits Migration, Invasion, and Angiogenesis of Human Non-Small Cell Lung Cancer A549 Cells by Inhibiting the FAK/Rho Signaling Pathway. Integrative Cancer Therapies, 10, 341-349. https://doi.org/10.1177/1534735410384860

[17] Katoh, K. (2017) Activation of Rho-Kinase and Focal Adhesion Kinase Regulates the Organization of Stress Fibers and Focal Adhesions in the Central Part of Fibroblasts. PeerJ, 5, e4063. https://doi.org/10.7717/peerj.4063

[18] Dobbins, K.R. and Saul, R.F. (2000) Transient Visual Loss after Licorice Ingestion. Journal of Neuro-Ophthalmology, 20, 38-41. https://doi.org/10.1097/00041327-200020010-00013

[19] Goel, B., Sharma, A., Tripathi, N., et al. (2020) In-Vitro Antitumor Activity of Compounds from Glycyrrhiza glabra against C6 Glioma Cancer Cells: Identification of Natural Lead for Further Evaluation. Natural Product Research, 1, 1-4. https://doi.org/10.1080/14786419.2020.1786830 
[20] Huttenlocher, A. and Horwitz, A.R. (2011) Integrins in Cell Migration. Cold Spring Harbor Perspectives in Biology, 3, a005074. https://doi.org/10.1101/cshperspect.a005074

[21] Parsons, J.T., Horwitz, A.R. and Schwartz, M.A. (2010) Cell Adhesion: Integrating Cytoskeletal Dynamics and Cellular Tension. Nature Reviews Molecular Cell Biology, 11, 633-643. https://doi.org/10.1038/nrm2957

[22] Vicente-Manzanares, M., Ma, X., Adelstein, R.S., et al. (2009) Non-Muscle Myosin II Takes Centre Stage in Cell Adhesion and Migration. Nature Reviews Molecular Cell Biology, 10, 778-790. https://doi.org/10.1038/nrm2786

[23] Conti, M.A. and Adelstein, R.S. (2008) Nonmuscle Myosin II Moves in New Directions. Journal of Cell Science, 121, 11-18. https://doi.org/10.1242/jcs.007112

[24] Stewart, S.A., Domínguez-Robles, J., Donnelly, R.F., et al. (2018) Implantable Polymeric Drug Delivery Devices: Classification, Manufacture, Materials, and Clinical Applications. Polymers-Basel, 10, 1379. https://doi.org/10.3390/polym10121379

[25] Wolinsky, J.B., Colson, Y.L. and Grinstaff, M.W. (2012) Local Drug Delivery Strategies for Cancer Treatment: Gels, Nanoparticles, Polymeric Films, Rods, and Wafers. Journal of Controlled Release, 159, 14-26. https://doi.org/10.1016/j.jconrel.2011.11.031

[26] DeSouza, R., Zahedi, P., Allen, C.J., et al. (2010) Polymeric Drug Delivery Systems for Localized Cancer Chemotherapy. Drug Delivery, 17, 365-375.

https://doi.org/10.3109/10717541003762854 